\title{
Keto analogues and amino acid supplementation and its effects on ammonaemia during extenuating endurance exercise in ketogenic diet-fed rats
}

\author{
Rogério T. Ferreira ${ }^{1,2,3}$, Sandra C. Gonçalves ${ }^{1,2,3}$, Maria L. Pedrosa ${ }^{2}$, Marcelo E. Silva ${ }^{3}$, Adriana Bassini ${ }^{1}$, \\ Wagner S. Coelho ${ }^{1,4}$, Aníbal M. de Magalhães-Neto ${ }^{1,5}$, Eduardo S. Prado ${ }^{6,7}$ and L. C. Cameron ${ }^{1_{*}}$ \\ ${ }^{1}$ Laboratory of Protein Biochemistry, Federal University of State of Rio de Janeiro, Rio de Janeiro, RJ 22290-255, Brazil \\ ${ }^{2}$ Laboratory of Metabolic Biochemistry, Department of Biological Science, Federal University of Ouro Preto, Ouro Preto, \\ MG 35400-000, Brazil \\ ${ }^{3}$ Laboratory of Experimental Nutrition, Department of Food, Federal University of Ouro Preto, Ouro Preto, \\ MG 35400-000, Brazil \\ ${ }^{4}$ Biological and Health Sciences Center, State University of West Zone Center, Rio de Janeiro, RJ 23070-200, Brazil \\ ${ }^{5}$ Biological and Health Sciences Institute, Federal University of Mato Grosso, Barra do Garças, MT 78600-000, Brazil \\ ${ }^{6}$ Laboratory for Research in Physical Exercise and Metabolism, Federal University of Alagoas, Alagoas, AL 57072-900, Brazil \\ ${ }^{7}$ Graduate Program in Nutrition, Federal University of Alagoas, Alagoas, AL 57072-900, Brazil
}

(Submitted 30 August 2017 - Final revision received 29 March 2018 - Accepted 8 June 2018 - First published online 6 August 2018 )

\section{Abstract}

Keto analogues and amino acids (KAAA) supplementation can reduce blood ammonia concentrations in athletes undergoing high-intensity exercise under both ketogenic and thermoneutral conditions. This study evaluated the acute effects of KAAA supplementation on ammonia metabolism during extenuating endurance exercise in rats fed a ketogenic diet. In all, eighty male Fischer rats at $90 \mathrm{~d}$ of age were divided into eight groups, and some were trained using a swimming endurance protocol. A ketogenic diet supplemented with keto analogues was administered for $10 \mathrm{~d}$. Administration of the ketogenic diet ended $3 \mathrm{~d}$ before the exhaustion test (extenuating endurance exercise). A ketogenic diet plus KAAA supplementation and extenuating endurance exercise (trained ketogenic diet supplemented with KAAA (TKKa)) increased blood ammonia concentrations by approximately $50 \%$ compared with the control diet (trained control diet supplemented with KAAA (TCKa)) and similar training (effect size $=1.33$; statistical power $=0.50$ ). The KAAA supplementation reduced blood urea concentrations by 4 and $18 \%$ in the control and ketogenic diet groups, respectively, compared with the groups fed the same diets without supplementation. The trained groups had $60 \%$ lower blood urate concentrations after TCKa treatment than after TKKa treatment. Our results suggest that KAAA supplementation can reduce blood ammonia concentrations after extenuating endurance exercise in rats fed a balanced diet but not in rats fed a ketogenic diet.

Key words: Metabolism: Low-carbohydrate diet: Exercise: Urate: Urea

An elevated concentration of ammonia in the blood (hyperammonaemia, above $300 \mu \mathrm{mol} / \mathrm{l}$ ) is toxic and has deleterious effects on the central nervous system, as observed in patients with hepatic disorders, mitochondrial dysfunction or oxidative stress in skeletal muscles ${ }^{(1-3)}$. Hyperammonaemia can also occur during exercise via both amino acid and AMP deamination in an intensity- and duration-dependent manner; urate is the final metabolite of inosine monophosphate (IMP), which is generated by AMP deamination ${ }^{(4)}$.

In addition, higher blood ammonia concentrations can result from a low-carbohydrate diet (termed a 'ketogenic diet'), and ammonia concentrations can be increased by both branchedchain amino acids oxidation and purine nucleotide metabo$\operatorname{lism}^{(5,6)}$. It has been suggested that hyperammonaemia may have a significant impact during intense endurance exercise under different environmental conditions and that it could induce neurological disturbances similar to the symptoms of hepatic encephalopathy, thus impairing athletic performance ${ }^{(7,8)}$. Therefore, it is believed that controlling the increase in blood ammonia concentration can improve exercise performance ${ }^{(4)}$.

Our group has been investigating ammonia metabolism in both humans and animals under exercise-induced stress in

Abbreviations: KAAA, keto analogues and amino acids; TC, trained control diet; TCKa, trained control diet supplemented with KAAA; TK, trained ketogenic diet; TKKa, trained ketogenic diet supplemented with KAAA; UC, untrained control diet; UCKa, untrained control diet supplemented with KAAA; UK, untrained ketogenic diet; UKKa, untrained ketogenic diet supplemented with KAAA.

* Corresponding author: L. C. Cameron, fax +55 212542 5280, email cameron@unirio.br 
combination with different diets ${ }^{(9-17)}$. In these studies, animals can be preferentially selected and assessed as good models to study general principles, and the results can then be compared with those obtained from humans. Recently, we demonstrated that keto analogues and amino acids (KAAA) supplementation can reduce blood ammonia concentrations during moderate prolonged exercise in athletes fed a ketogenic diet under thermoneutral conditions ${ }^{(18)}$ and in rats during resistance exercise ${ }^{(9)}$. In both cases, we postulate that KAAA supplementation increases glucose availability for exercise through gluconeogenesis. Diets are known to modify metabolic responses, especially during exercise $^{(6)}$, and here, we present new information on the metabolic differences associated with KAAA supplementation in association with two different diets.

To the best of our knowledge, information is not available on the effects of KAAA supplementation on ammonia metabolism after high-intensity exercise in rats fed a ketogenic diet. We hypothesised that KAAA supplementation reduces blood ammonia concentrations under these conditions. Therefore, in the present study, we evaluated the acute effects of KAAA supplementation on ammonia metabolism during extenuating endurance exercise in rats fed a ketogenic diet.

\section{Methods \\ Animals}

Male Fischer rats ( $90 \mathrm{~d}$ of age, with an initial and final body mass of 203.9 (SE 0.9) and 382.5 (SE 29.9) g, respectively) were randomly divided into eight groups of ten animals each as follows: untrained control diet (UC); untrained ketogenic diet (UK); untrained control diet supplemented with KAAA (UCKa); untrained ketogenic diet supplemented with KAAA (UKKa); trained control diet (TC); trained ketogenic diet (TK); trained control diet supplemented with KAAA (TCKa) and trained ketogenic diet supplemented with KAAA (TKKa). The animals were kept in individual cages under controlled environmental conditions $\left(23 \pm 1^{\circ} \mathrm{C}, 55 \pm 10\right) \%$ relative humidity, $12 \mathrm{~h}$ dark$12 \mathrm{~h}$ light cycle). Rats showing abnormal behaviour or rapid weight loss were excluded. This study followed the animal care procedures regulated by the Brazilian College of Animal Experimentation, and the study protocol was submitted to and approved by the Ethics Committee of the Ouro Preto Federal University (036/2008).

\section{Procedures}

The groups supplemented with KAAA received $0 \cdot 1 \mathrm{~g}$ Ketosteri ${ }^{\circledR}$ (Fresenius Kabi) in $2.0 \mathrm{ml}$ of water $(0.3 \mathrm{~g} / \mathrm{kg})$. The composition of the KAAA mixture per tablet was as follows: $\alpha$-keto analogues of isoleucine $(335 \mathrm{mg})$, leucine $(505 \mathrm{mg})$, phenylalanine ( $430 \mathrm{mg}$ ) and valine $(340 \mathrm{mg}$ ); an $\alpha$-hydroxy analogue of methionine $(295 \mathrm{mg})$; L-lysine acetate $(75 \mathrm{mg})$; L-threonine $(265 \mathrm{mg})$; L-tryptophan (115 mg); L-histidine (190 mg) and L-tyrosine (150 mg). All other groups received $2.0 \mathrm{ml}$ of $0.9 \% \mathrm{NaCl}$ orally $1 \mathrm{~h}$ before exercise and served as control groups.

The trained groups received training that consisted of an adapted version of the swimming protocol used in an animal
Table 1. Nutritional composition of diets $(\mathrm{g} / 1000 \mathrm{~g})$

\begin{tabular}{lcc}
\hline Ingredients & AIN-93M* & Ketogenic ${ }^{*}$ \\
\hline Vitamins, mixed & $10 \cdot 0$ & $10 \cdot 0$ \\
Choline & $2 \cdot 5$ & - \\
Minerals, mixed & $35 \cdot 0$ & $35 \cdot 0$ \\
Fibres & $50 \cdot 0$ & $50 \cdot 0$ \\
Sucrose & $100 \cdot 0$ & - \\
Casein & $141 \cdot 8$ & $200 \cdot 0$ \\
Maize starch & $620 \cdot 7$ & - \\
Soya oil & $40 \cdot 0$ & - \\
Maize oil & - & $102 \cdot 0$ \\
Lard & - & $424 \cdot 7$ \\
Margarine & - & 178.3 \\
Total & 1000 & 1000 \\
\hline
\end{tabular}

KAAA, keto analogues and amino acids.

* Standard/control diet AIN-93M ${ }^{(21)}$. Untrained control diet, untrained control diet supplemented with KAAA, trained control diet, trained control diet supplemented with KAAA groups.

$\dagger$ Ketogenic diet ${ }^{(20)}$. Untrained ketogenic diet, untrained ketogenic diet supplemented with KAAA, trained ketogenic diet, trained ketogenic diet supplemented with KAAA groups.

study conducted by Gobatto et $a l .{ }^{(19)}$. The animals were trained for $30 \mathrm{~min} 5 \mathrm{~d}$ a week over a $79-\mathrm{d}$ period. Training began at the same time each day (08.30 hours), and the water temperature was maintained at $31 \pm 1^{\circ} \mathrm{C}$. During the first $7 \mathrm{~d}$, the animals were allowed to adapt to the training protocol by incrementally increasing the water level daily until they were no longer able to touch the bottom of the tank with their tail. On days 8 through 78 , the load was incrementally increased by $1 \%$ of the animal's body mass by attaching a load to the animal's back. Furthermore, on days 8 through 69 of training, all animals received a balanced rodent diet (Labina ${ }^{\circledR}$; Purina). The groups were then randomly divided. On days 70 through 79 , the animals received either the ketogenic ${ }^{(20)}$ or control ${ }^{(21)}$ diet ad libitum during the course of the protocol (Table 1). On the 80th day, the training protocol was administered to promote extenuating endurance exercise according to previously described methods ${ }^{(22)}$. The exhaustion test (termed 'extenuating exercise') was conducted using a load equal to $15 \%$ of the animal's body mass. In addition, on the 80th day, blood, hepatic and muscle glycogen concentrations were determined (Fig. 1(a)).

To ensure the ketogenic effects, the amount of ketone bodies in urine was assessed every $3 \mathrm{~d}$ through ketonuria analysis with qualitative urinalysis reagent strips ${ }^{(23)}$. Body weight and feeding behaviour were measured weekly. Rats were divided into eight groups (Fig. 1(b)), but KAAA supplementation (Ketosteril ${ }^{\circledR}$; Fresenius Kabi) was administered $3 \mathrm{~d}$ before testing in the following groups: UCKa, UKKa, TCKa and TKKa. The supplement was administered via gavage at 19.30 hours ( $\pm 60 \mathrm{~min}$ ) every day before training.

On the 80th day, all animals were fasted (about 10.00 hours), anaesthetised with isoflurane and euthanised via bleeding at the brachial plexus ${ }^{(24)}$. Blood was immediately collected and centrifuged (14000 $\boldsymbol{g}$ for $15 \mathrm{~min}$ ) to obtain either plasma (in a vacuum tube with the anticoagulant EDTA for ammonia measurements) or serum (in a vacuum tube without anticoagulant for urea, urate, creatinine, glucose and lactate measurements), which was aliquoted and stored in liquid nitrogen for no more than $3 \mathrm{~d}$ before biochemical analysis. Analyses were performed 
(a)

\begin{tabular}{|c|c|c|c|c|c|c|}
\hline $\begin{array}{c}\text { Animal's } \\
\text { adaptation } \\
\downarrow\end{array}$ & $\begin{array}{c}\text { Balanced food } \\
\downarrow\end{array}$ & $\begin{array}{c}\text { Diets } \\
\text { control and } \\
\text { ketogenic } \\
\downarrow\end{array}$ & $\begin{array}{l}\text { Supp } \\
\downarrow\end{array}$ & $\begin{array}{c}\text { lemen } \\
+ \\
\text { diets } \\
\downarrow\end{array}$ & $\begin{array}{l}\text { ation } \\
\downarrow\end{array}$ & $\begin{array}{c}\text { Collection } \\
\downarrow\end{array}$ \\
\hline 1 st up to 7 th day & 8th up to 69 th day & 70 th up to & 77 th & 78th & 79th & 80th day \\
\hline
\end{tabular}

(b)

g. 1. Experimental design and timeline. Animal adaptation, training protocol, diets, supplementation and collection (a). Rats were divided into eight groups (b). UC, untrained control diet; UK, untrained ketogenic diet; UCKa, untrained control diet supplemented with KAAA; UKKa, untrained ketogenic diet supplemented with KAAA; TC, trained control diet; TK, trained ketogenic diet; TCKa, trained control diet supplemented with KAAA; TKKa, trained ketogenic diet supplemented with KAAA.

using commercially available spectrophotometric assays (Labtest and Randox) using enzyme kinetic-based methods on a Dade Model Dimension RXL Automated Chemistry Analyzer (Dade Behring). Hepatic and muscle glycogen concentrations were examined in rats according to previously described methods ${ }^{(25,26)}$. In brief, tissue was sliced and homogenised in 5\% TCA. The homogenate was centrifuged at $14000 \mathbf{g}$, and the supernatant was extracted with ethanol (1:1). The mixture was centrifuged at $14000 \mathbf{g}$, the supernatant was mixed with acetic acid (1:1), and the mixture was centrifuged again. The supernatant was brought to $\mathrm{pH} 7.4$ and analysed with a colorimetric glucose oxidase activity kit (Labtest Diagnostica).

\section{Statistical analyses}

Statistically significant differences in the data were assessed using one-way ANOVA and the post hoc Holm-Sidak test. Corresponding non-parametric tests were used to analyse data with non-normal distributions. A value of $P<0.05$ indicated statistical significance for all comparisons. Data are presented as the mean values with their standard errors. Statistical analyses were performed using SigmaStat version 3.5 for Windows (Systat Software Inc.). Sample size, Cohen's $d$ effect size and statistical power were calculated to analyse data and identify statistically significant differences in the concentrations of hepatic glycogen, skeletal muscle glycogen and blood ammonia and urate. In this case, the thresholds for small, medium and large effects were 0.20, 0.50 and $0 \cdot 80$, respectively. Sample size, Cohen's $f$ effect size and statistical power were also calculated to analyse data and identify statistically significant differences in blood urea, creatinine, glucose and lactate concentrations by performing a post hoc analysis (G*Power software version 3.1.9.2, University of Kiel). Here, the thresholds for small, medium and large effects were $0 \cdot 10,0 \cdot 25$ and $0 \cdot 40$, respectively ${ }^{(27)}$. Complete sample statistical analyses are shown in the online Supplementary Material.

\section{Results}

The ketogenic diet group tested positive for ketosis after the 3rd day on the diet (data not shown). During exercise, both groups were maintained under controlled temperature conditions (approximately $31^{\circ} \mathrm{C}$ ). The untrained and trained groups had similar times to exhaustion at the same exercise intensity (Fig. 2).

At rest, the hepatic glycogen concentration was $10 \cdot 6$ (sE 0.2) $\mu \mathrm{g} / \mathrm{g}$. Significant differences between the untrained groups (UC $v$. UK) were not observed. However, KAAA supplementation reduced the hepatic glycogen concentration by approximately $30 \%$ (UCKa $v$. UC; effect size $=2 \cdot 47$; statistical power $=0 \cdot 99$ ). Furthermore, the ketogenic diet plus KAAA supplementation decreased the glycogen concentration to approximately $50 \%$ compared with the control diet (UKKa $v$. UC; effect size $=3 \cdot 97$; statistical power $=1 \cdot 0$ ). Extenuating endurance exercise did not reduce the hepatic glycogen concentration in rats fed the control diet (TC $v$. UC; effect size $=0 \cdot 07$; statistical power $=0 \cdot 05$ ). Compared with the untrained group, the trained group fed the ketogenic diet experienced a decrease in hepatic glycogen concentration by approximately $22 \%$ (TK $v$. TC; effect size $=$ 

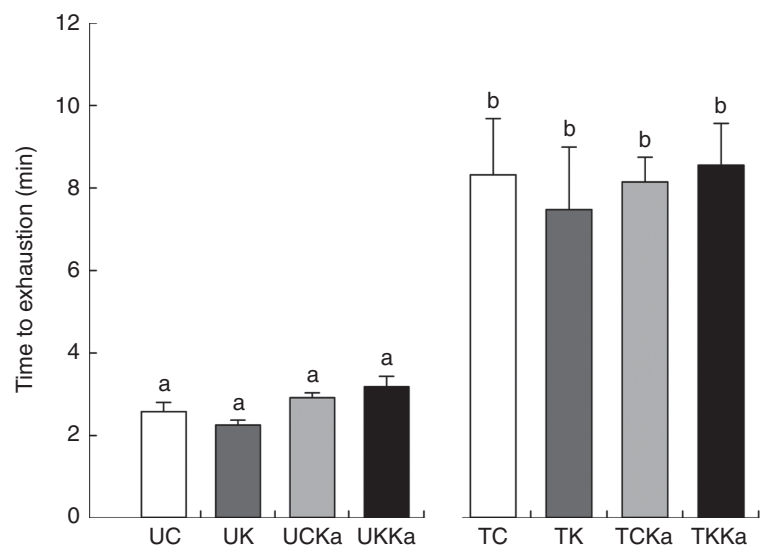

Fig. 2. Time to exhaustion after extenuating endurance exercise. Time to exhaustion in the last test is presented as mean values with their standard errors for all groups according to the experimental design. Untrained and trained groups are separated by a break. KAAA, keto analogues and amino acids, UC, untrained control diet; UK, untrained ketogenic diet; UCKa untrained control diet supplemented with KAAA; UKKa, untrained ketogenic diet supplemented with KAAA; TC, trained control diet; TK, trained ketogenic diet; TCKa, trained control diet supplemented with KAAA; TKKa, trained ketogenic diet supplemented with KAAA. ${ }^{a, b}$ Mean values with unlike letters were significantly different $(P<0.05)$.

1.35; statistical power $=0 \cdot 67$ ). The KAAA supplementation in the trained groups led to lower hepatic glycogen concentrations in both groups (TCKa and TKKa) than in the trained control group ( $43 \%$ for TCKa $v$. TC; effect size $=1 \cdot 86$; statistical power $=0.91$; and $36 \%$ for TKKa $v$. TC; effect size $=1 \cdot 75$; statistical power $=$ 0.88) (Fig. 3(A)). The total hepatic glycogen concentration did not change in any of the groups (10.4 (SE 0.4) $\mu \mathrm{g} / \mathrm{g}$ ).

The skeletal muscle glycogen concentration was 42.7 (SE 1.15$) \mu \mathrm{g} / \mathrm{g}$ during rest but was reduced by the ketogenic diet. Diet manipulation in the untrained groups did not affect the skeletal muscle glycogen concentration in response to exercise. However, training did increase the skeletal muscle glycogen concentration by $12.5 \%$ (TC $v$. UC; effect size $=1.03$; statistical power $=0.53)$ and $22.8 \%$ (TK $v$. UK; effect size $=$ 1.34 ; statistical power $=0.72$ ). In addition, KAAA supplementation plus the control diet increased the skeletal muscle glycogen concentration in untrained rats by $41 \%$ (UCKa $v$. UC; effect size $=3 \cdot 15$; statistical power $=0.99)$ and in trained rats by $36 \%($ TCKa $v$. TC; effect size $=1.44$; statistical power $=0.73)$ (Fig. 3(B)).

To investigate the effects of KAAA supplementation on ammonia metabolism, we measured blood ammonia concentrations after swimming. The blood ammonia concentration was 59.4 ( $\mathrm{se} 21.4) \mu \mathrm{mol} / \mathrm{l}$ at baseline and decreased by $5 \%$ in response to acute exercise after training in supplemented animals compared with untrained animals fed the same diet (TC $v$. UC; effect size $=0.28$; statistical power $=0.07$ ). The blood ammonia concentration was lower after exercise in the untrained group fed a ketogenic diet (approximately 20\%) than in the trained group fed the same diet (TK) (effect size $=0.62$; statistical power $=0 \cdot 19$ ). There were no differences in the blood ammonia concentration between the untrained group supplemented with KAAA and the untrained group fed the control diet (UCKa $v$. UC; effect size $=0 \cdot 76 ;$ statistical power $=0 \cdot 29$ ).
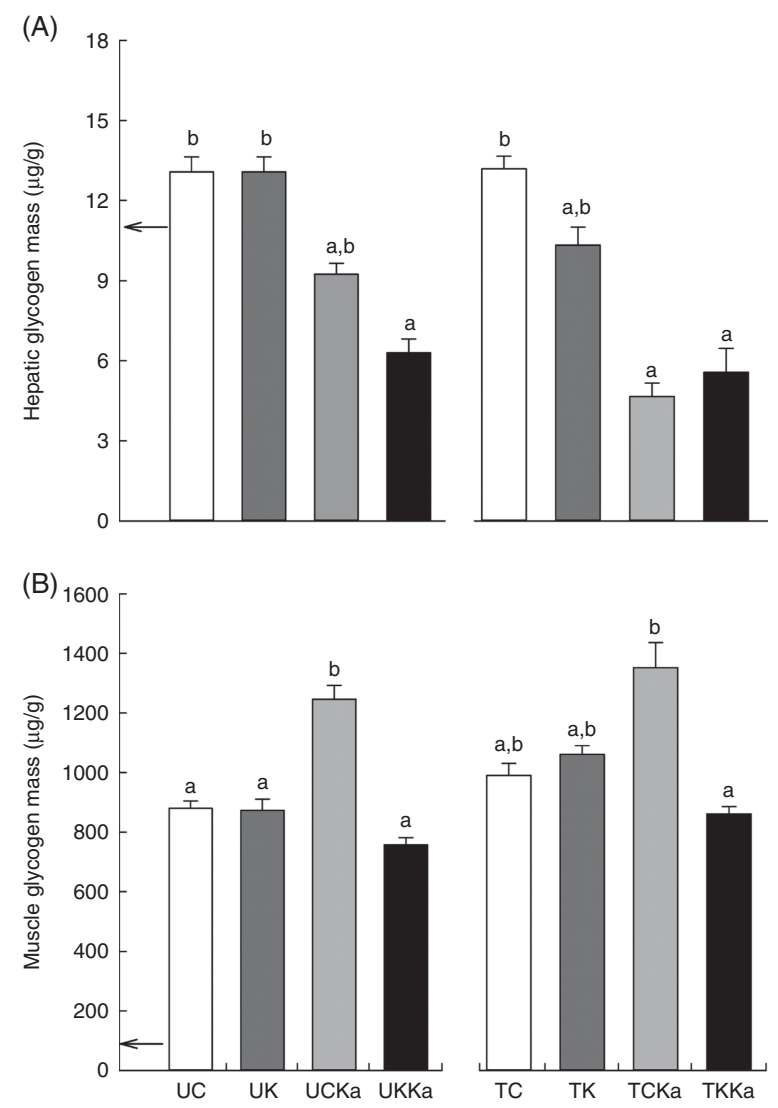

Fig. 3. Hepatic and muscle glycogen concentrations after extenuating endurance exercise presented as mean values with their standard errors. Hepatic (A) and muscle (B) glycogen concentrations. KAAA, keto analogues and amino acids, UC, untrained control diet; UK, untrained ketogenic diet; UCKa, untrained control diet supplemented with KAAA; UKKa, untrained ketogenic diet supplemented with KAAA; TC, trained control diet; TK, trained ketogenic diet; TCKa, trained control diet supplemented with KAAA; TKKa, trained ketogenic diet supplemented with KAAA; $\longleftarrow$, mean basal hepatic and muscle glycogen concentrations. ${ }^{a, b}$ Mean values with unlike letters were significantly different $(P<0.05)$.

Therefore, KAAA did not affect blood ammonia concentrations in untrained groups.

Interestingly, the blood ammonia concentration increased by $37 \%$ in animals fed a ketogenic diet with KAAA supplementation compared with those fed the control diet (UKKa $v$. UC; effect size $=1 \cdot 14$; statistical power $=0 \cdot 56$ ). Diet did not affect the blood ammonia concentration in the untrained or trained groups (UC $v$. UK; effect size $=0.31$; statistical power $=0.08$; and TC $v$. TCKa; effect size $=0 \cdot 44$; statistical power $=0.08)$. In the trained groups, the ketogenic diet plus KAAA supplementation and extenuating endurance exercise increased the blood ammonia concentration by approximately 50\% compared with the control diet (TKKa $v$. TCKa; effect size $=1 \cdot 33$; statistical power $=0 \cdot 50$ ), suggesting that blood ammonia concentrations are higher after exercise and KAAA supplementation (Fig. 4(A)).

To evaluate the effect of KAAA supplementation on urea metabolism, we measured blood urea concentrations. The resting blood urea concentration was equivalent in all groups $(7.8(\mathrm{SE} \quad 0 \cdot 2) \mathrm{mmol} / \mathrm{l})$ and did not differ between the two untrained groups fed either the control or ketogenic diet 


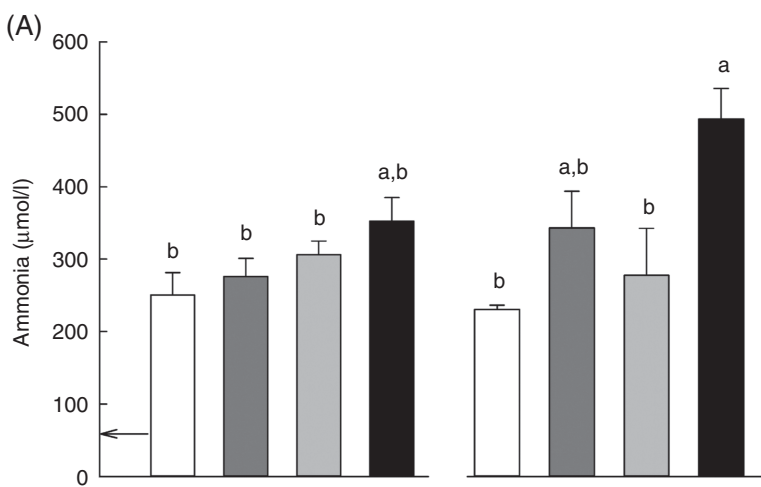

(B)

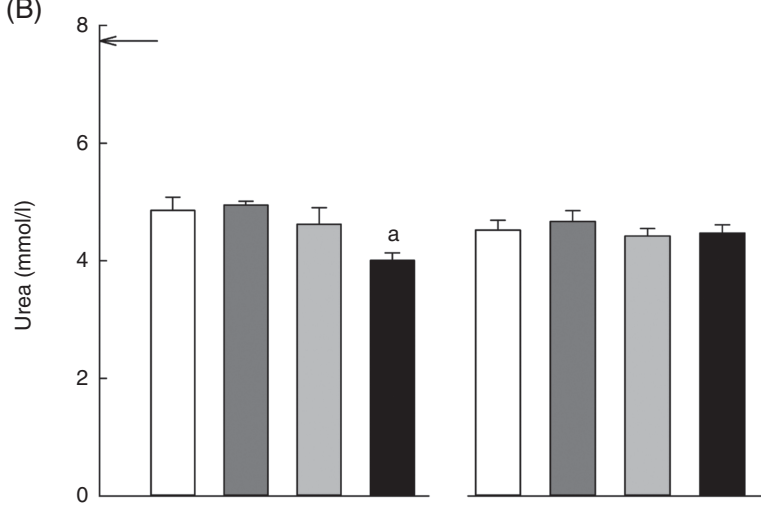

(C)

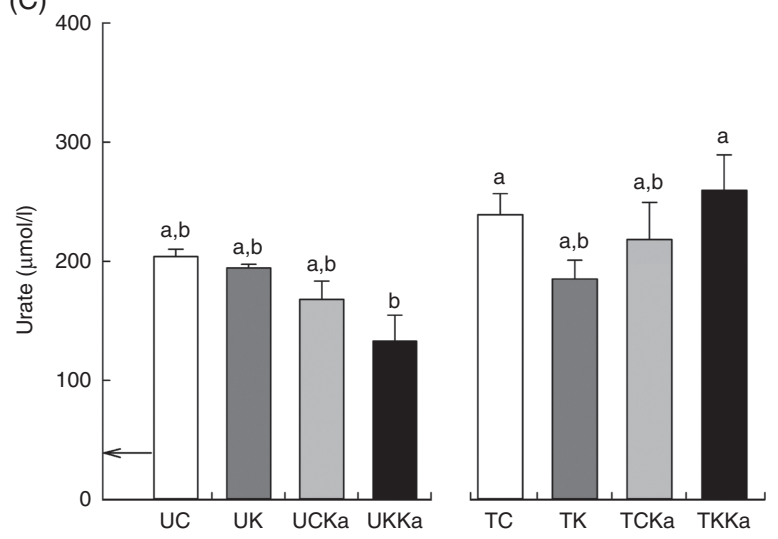

Fig. 4. Keto analogues and amino acids (KAAA) supplementation and a ketogenic diet increase blood ammonia and urate concentrations after extenuating endurance exercise presented as mean values with their standard errors. Blood ammonia (A), urea (B) and urate (C) concentrations. UC, untrained control diet; UK, untrained ketogenic diet; UCKa, untrained control diet supplemented with KAAA; UKKa, untrained ketogenic diet supplemented with KAAA; TC, trained control diet; TK, trained ketogenic diet; TCKa, trained control diet supplemented with KAAA; TKKa, trained ketogenic diet supplemented with KAAA; $\longleftarrow$, mean basal metabolite levels. ${ }^{a, b}$ Mean values with unlike letters were significantly different $(P<0.05)$.

(UC $v$. UK). However, KAAA supplementation significantly deceased the blood urea concentration in the untrained group fed a ketogenic diet (effect size $=0 \cdot 50$; statistical power $=0 \cdot 77$ ). Differences were not observed between the trained groups (Fig. 4(B)).

To differentiate ammonia produced from AMP deamination from that produced from amino acid deamination, we measured blood urate concentrations. The basal blood urate concentration was 37.6 (se 1.8) $\mu \mathrm{mol} / \mathrm{l}$. Independent of training, KAAA supplementation significantly reduced the blood urate concentration (approximately 30\%) in the UKKa group compared with the UC group (effect size $=1 \cdot 26$; statistical power $=0 \cdot 68$ ) . This effect in the supplemented group was enhanced after extenuating endurance exercise. The blood urate concentration was significantly higher in the TKKa group than in the TCKa group (effect size $=0 \cdot 60$; statistical power $=0 \cdot 13)($ Fig. $4(\mathrm{C})$ ).

The basal blood creatinine concentration was $30 \cdot 0$ (sE 1.5$) \mathrm{mmol} / \mathrm{l}$. Decreases in blood creatinine concentration were only observed in the UC and untrained groups. Despite decreases in the TC group, blood creatinine concentrations did not change in the TKKa, TCKa and TK groups (effect size $=$ 0.59; statistical power $=0.92)$ (Fig. 5(A)).

To understand the effect of KAAA supplementation on glucose maintenance, we measured glucose concentrations in all of the groups. The basal glucose concentration was 9.1 (sE 0.3 ) $\mathrm{mmol} / \mathrm{l}$, and we did not detect significant differences within the groups (effect size $=0.39$; statistical power $=0.59$ ) (Fig. 5(B)).

Blood lactate concentration is an indicator of glucose metabolism during exercise. The basal lactate concentration was $4 \cdot 0$ (sE $0 \cdot 3$ ) $\mathrm{mmol} / \mathrm{l}$. Significant KAAA-induced differences were not observed between the TCKa $(7.4(\operatorname{se~} 0.01) \mathrm{mmol} / \mathrm{l})$ and TKKa $(7.4(\mathrm{SE} 0 \cdot 2) \mathrm{mmol} / \mathrm{l})$ groups or between the UKKa $(7 \cdot 7$ $(\mathrm{sE} 0.7) \mathrm{mmol} / \mathrm{l})$ and UCKa $(7.4(\mathrm{sE} 0.03) \mathrm{mmol} / \mathrm{l})$ groups after extenuating endurance exercise. However, blood lactate concentration was increased in the groups supplemented with KAAA compared with the unsupplemented groups with or without a ketogenic diet after extenuating endurance exercise $($ effect size $=0 \cdot 87$; statistical power $=0 \cdot 99)($ Fig. $5(\mathrm{C}))$.

\section{Discussion}

The aim of this study was to investigate the acute effects of KAAA supplementation on ammonia metabolism during extenuating endurance exercise in rats fed a ketogenic diet. Here, we used a ketogenic diet and extenuating endurance exercise to decrease glycogen availability in liver and muscle, increasing the need for amino acids as an energy source and inducing hyperammonaemia ${ }^{(28-30)}$. Our results indicated that KAAA supplementation reduced the blood ammonia concentration during extenuating endurance exercise in rats fed a balanced diet; however, the ammonia concentration was unaffected in rats fed a ketogenic diet.

The KAAA supplementation has been widely used to treat patients with chronic kidney disease and hepatic disorders, such as hepatic encephalopathy and hyperammonaemia ${ }^{(31,32)}$. However, it is also known that carbohydrate ingestion may attenuate blood ammonia concentrations during prolonged submaximal exercise by reducing amino acid degradation ${ }^{(5)}$. In addition, glycogen depletion in muscles could lead to a more rapid increase in blood ammonia concentration ${ }^{(33)}$. In addition, high energy demand and protein catabolism can exacerbate exerciseinduced hyperammonaemia in humans and animals ${ }^{(34-36)}$. Previous work from our lab demonstrated that during exercise, 

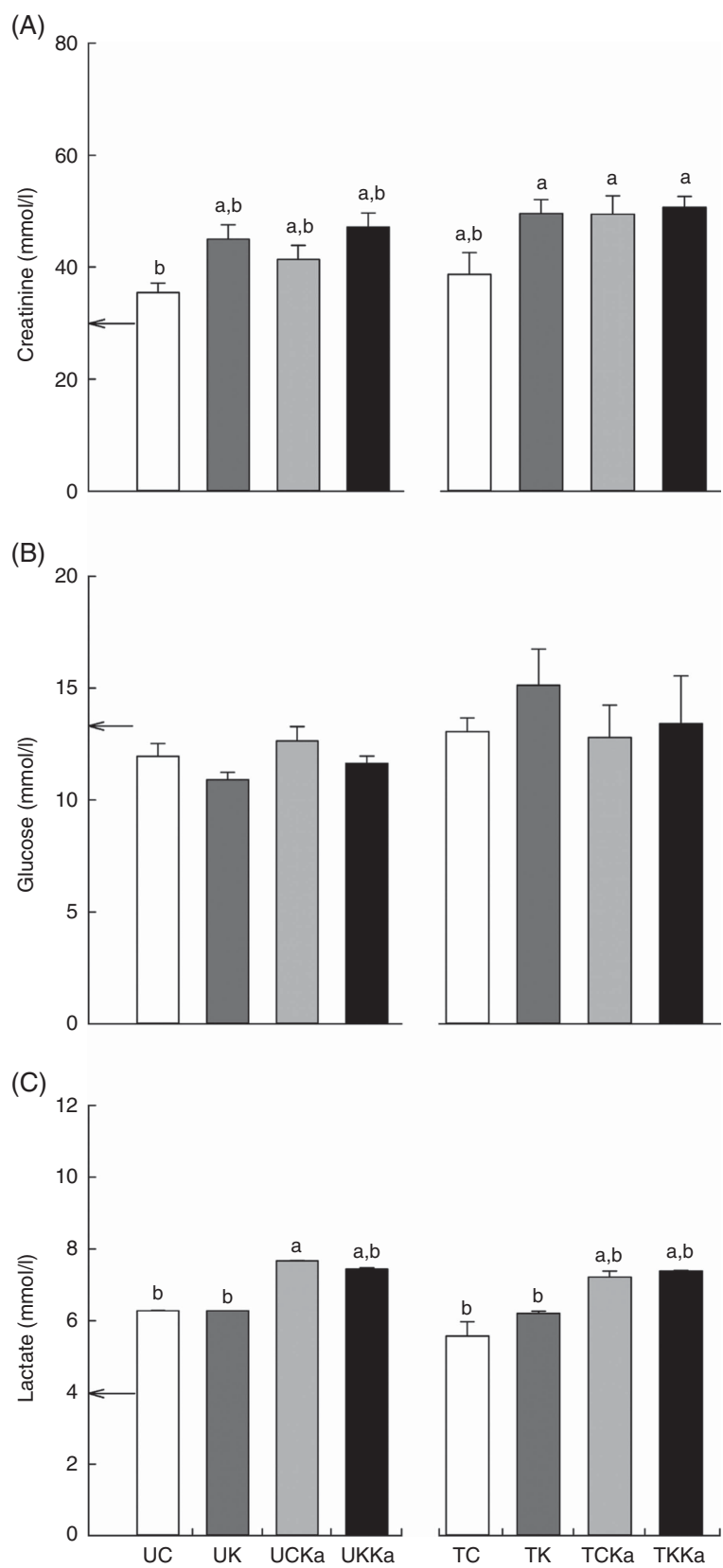

Fig. 5. Keto analogues and amino acids (KAAA) supplementation and a ketogenic diet affect blood lactate concentrations but not creatinine and glucose concentrations after extenuating endurance exercise. Results are presented as mean values with their standard errors. Blood creatinine (A), glucose (B) and lactate (C) concentrations. UC, untrained control diet; UK, untrained ketogenic diet; UCKa, untrained control diet supplemented with KAAA; UKKa, untrained ketogenic diet supplemented with KAAA; TC, trained control diet; TK, trained ketogenic diet; TCKa, trained control diet supplemented with KAAA; TKKa, trained ketogenic diet supplemented with KAAA; $\longleftarrow$, mean basal metabolite levels. ${ }^{\mathrm{a}, \mathrm{b}}$ Mean values with unlike letters were significantly different $(P<0.05)$.

KAAA supplementation decreases the concentrations of ammonia, urea and urate in the plasma of humans fed a ketogenic diet ${ }^{(12)}$ and rats fed a non-ketogenic diet ${ }^{(9)}$. The results of this study demonstrated that KAAA supplementation significantly reduced the blood ammonia concentration in trained rats fed the balanced control diet, which may have occurred by increasing the muscle glycogen concentration. We can explain this finding by a synergistic effect of carbohydrate ingestion and the use of KAAA for glycogen synthesis.

High-intensity exercise is known to increase ammonia production via AMP deamination ${ }^{(37)}$. A previous study demonstrated that higher blood concentrations of catecholamines and IMP are produced when training exercise is continued until exhaustion ${ }^{(38)}$. Furthermore, a low-carbohydrate diet has been found to increase the blood ammonia concentration and blood noradrenaline and adrenaline concentrations during exercise ${ }^{(6)}$. The trained group fed the ketogenic diet had a lower muscle glycogen concentration than the trained group fed a balanced diet. Supplementation with KAAA did not decrease ammonaemia in the group fed a ketogenic diet. It is possible that these results are due to extenuating endurance exercise or to effects of environmental temperature during exercise ${ }^{(8)}$.

In addition, exercise in hot conditions appears to exacerbate exercise-induced hyperammonaemia ${ }^{(34)}$. The rats were subjected to a swimming protocol to the point of exhaustion in a hot environment (approximately $31^{\circ} \mathrm{C}$ ). In humans, Mohr et al. ${ }^{(39)}$ demonstrated that blood ammonia concentrations were higher during intermittent exercise performed under heat stress conditions than under thermoneutral conditions. Increased intramuscular ammonia accumulation has been found to occur in both endurance-trained and untrained humans during heat stress. This accumulation is likely the result of an increasing concentration of IMP, a product of AMP deamination ${ }^{(40)}$. Furthermore, blood adrenaline and noradrenaline concentrations have been shown to be higher during exercise under heat stress conditions $^{(41)}$. We previously showed that under thermoneutral conditions, blood ammonia concentrations decreased in rats fed a standard diet and supplemented with KAAA after resistance exercise $^{(9)}$. In this study, the effects on blood ammonia concentration were greater under conditions of training at elevated temperature than at ambient temperature.

We measured blood urea concentrations to evaluate the effects of KAAA supplementation on urea metabolism in rats fed a ketogenic diet under extenuating endurance exercise. Although a previous study reported that a low protein diet and KAAA supplementation could reduce blood urea concentrations in patients with chronic renal failure disease without exercise $^{(42)}$, in our study, KAAA supplementation maintained uraemia after extenuating endurance exercise independent of diet. In addition, KAAA supplementation efficiently reduced uraemia in animals undergoing resistance exercise ${ }^{(9)}$. We can explain the discrepant results because the previous study was performed under fatiguing exercise conditions, not under extenuating endurance exercise.

Previous studies have reported that the ATP:ADP ratio decreases in muscle, leading to increased AMP deamination and ammonia production during low energetic states and extenuating exercise, such as in the current study ${ }^{(43)}$. In the present study, we observed that KAAA administration reduced basal urataemia. In addition, KAAA supplementation delayed the increase in urate concentration in the blood after extenuating exercise in trained animals fed the control diet. However, urataemia increased after extenuating exercise in trained animals 
that received KAAA supplementation and the ketogenic diet. Our data suggest that the increase in the blood ammonia concentration observed after extenuating exercise in the KAAAsupplemented group fed a ketogenic diet was primarily because of AMP deamination and not amino acid catabolism. These data suggest that heat stress could exacerbate adenine degradation ${ }^{(34,39,44)}$; however, the trained group fed a ketogenic diet had lower muscle glycogen concentrations than the trained group fed a balanced diet.

The KAAA supplementation has been used to treat patients with chronic kidney disease ${ }^{(31)}$. We evaluated blood creatinine concentrations in response to acute KAAA supplementation after extenuating exercise in rats fed different diets. Blood creatinine concentrations are influenced by diet and exercise $^{(45)}$ and can be used to evaluate renal function ${ }^{(46)}$. In this study, blood creatinine concentrations were similar in all of the groups supplemented with KAAA, suggesting similar kidney filtration rates across these groups. It is important to emphasise that we did not measure urinary creatinine in this study, but we demonstrated previously that KAAA supplementation increases creatinine clearance after resistance exercise $^{(9)}$; however, these data were acquired under thermoneutral environmental conditions and not under heat as in the current study. Blood creatinine concentrations were similarly increased in the exercise groups with and without heat stress $^{(35)}$

Consistent with our previous findings in humans ${ }^{(18)}$, KAAA supplementation maintained similar blood glucose concentrations in all the studied groups, including the ketogenic and control diet groups, after extenuating exercise. The KAAA supplement is a mixture of ketogenic and glucogenic KAAA that can be used to produce glucose via gluconeogenesis during exercise under thermoneutral environmental conditions $^{(9)}$. In the current study, KAAA supplementation exacerbated blood lactate concentrations after extenuating exercise in the trained groups with and without a ketogenic diet compared with the unsupplemented control group. In humans, blood lactate levels were found to be significantly higher under heat stress conditions than under thermoneutral environmental conditions after $5 \mathrm{~min}$ of high-intensity exercise, although this increase was not observed at any other time points during exercise ${ }^{(34)}$.

It is important to emphasise that body temperature was not measured in this study, and it may have limited our interpretations. Body temperature could have changed, and this parameter should be evaluated in similar studies in the future. This study demonstrated that extenuating endurance exercise and a ketogenic diet in combination with low glycogen stores and possibly heat stress exceed the protection offered by KAAA supplementation against hyperammonaemia under thermoneutral conditions.

In conclusion, we showed that KAAA supplementation could reduce blood ammonia concentrations in rats fed a control diet but not in rats fed a ketogenic diet after extenuating endurance exercise, which suggests that changes in ammonia metabolism differ under maximal voluntary effort and exhaustive exercise.

\section{Acknowledgements}

This study was funded by Comitê Olimpíco do Brasil; Conselho Nacional de Desenvolvimento Científico e Tecnológico; Coordenação de Aperfeiçoamento de Pessoal de Nível Superior; Federal University of Ouro Preto; Financiadora de Estudos e Projetos; Fundação Carlos Chagas Filho de Amparo à Pesquisa do Estado do Rio de Janeiro; Merck-Sigma-Aldrich; Universidade Federal do Estado do Rio de Janeiro and Waters Corporation.

L. C. C., M. L. P. and M. E. S. conceived and designed the experiment; R. T. F. and S. C. G. performed the experiment; M. L. P., M. E. S., A. B., W. S. C., E. S. P., A. M. d. M. and L. C. C. analysed the data; M. L. P. and M. E. S. contributed reagents/ materials/analysis tools; W. S. C., E. S. P. and L. C. C. wrote the paper.

The authors declare that there are no conflicts of interest.

\section{Supplementary material}

For supplementary material/s referred to in this article, please visit https://doi.org/10.1017/S0007114518001770

\section{References}

1. Felipo V \& Butterworth RF (2002) Neurobiology of ammonia. Prog Neurobiol 67, 259-279.

2. Stewart CA \& Cerhan J (2005) Hepatic encephalopathy: a dynamic or static condition. Metab Brain Dis 20, 193-204.

3. Davuluri G, Allawy A, Thapaliya S, et al. (2016) Hyperammonemia induced skeletal muscle mitochondrial dysfunction results in cataplerosis and oxidative stress. J Physiol $\mathbf{5 9 4}$ $7341-7360$

4. Wilkinson DJ, Smeeton NJ \& Watt PW (2010) Ammonia metabolism, the brain and fatigue; revisiting the link. Prog Neurobiol 91, 200-219.

5. Wagenmakers AJ, Beckers EJ, Brouns F, et al. (1991) Carbohydrate supplementation, glycogen depletion, and amino acid metabolism during exercise. Am J Physiol 260, E883-E890.

6. Langfort J, Czarnowski D, Zendzian-Piotrowska M, et al. (2004) Short-term low-carbohydrate diet dissociates lactate and ammonia thresholds in men. J Strength Cond Res 18, 260-265.

7. Banister EW \& Cameron BJ (1990) Exercise-induced hyperammonemia: peripheral and central effects. Int J Sports Med 11, S129-S142.

8. Nybo L (2010) CNS fatigue provoked by prolonged exercise in the heat. Front Biosci (Elite Ed) 2, 779-792.

9. de Almeida RD, Prado ES, Llosa CD, et al. (2010) Acute supplementation with keto analogues and amino acids in rats during resistance exercise. Br J Nutr 104, 1438-1442.

10. Carvalho-Peixoto J, Alves RC \& Cameron LC (2007) Glutamine and carbohydrate supplements reduce ammonemia increase during endurance field exercise. Appl Physiol Nutr Metab 32, 1186-1190

11. Bassini-Cameron A, Monteiro A, Gomes A, et al. (2008) Glutamine protects against increases in blood ammonia in football players in an exercise intensity-dependent way. Br J Sports Med 42, 260-266.

12. Prado ES, de Rezende Neto JM, de Almeida RD, et al. (2011) Keto analogue and amino acid supplementation affects the ammonaemia response during exercise under ketogenic conditions. Br J Nutr 105, 1729-1733. 
13. Bassini A, Magalhaes-Neto AM, Sweet E, et al. (2013) Caffeine decreases systemic urea in elite soccer players during intermittent exercise. Med Sci Sports Exerc 45, 683-690.

14. Goncalves LC, Bessa A, Freitas-Dias R, et al. (2012) A sportomics strategy to analyze the ability of arginine to modulate both ammonia and lymphocyte levels in blood after highintensity exercise. J Int Soc Sports Nutr 9, 30.

15. Bassini A \& Cameron LC (2014) Sportomics: building a new concept in metabolic studies and exercise science. Biochem Biophys Res Commun 445, 708-716.

16. Prado E, Souza GHMF, Pegurier M, et al. (2017) Non-targeted sportomics analyses by mass spectrometry to understand exercise-induced metabolic stress in soccer players. Int J Mass Spectrom 418, 1-5.

17. Lima RCP, Camerino SRAS, França TCL, et al. (2017) Keto analogues and amino acid supplementation induces decrease of white blood cell counts and muscle damage during intense exercise under thermoneutral conditions. Food Funct 8, 1519-1525.

18. Camerino SR, Lima RC, Franca TC, et al. (2016) Keto analogue and amino acid supplementation and its effects on ammonemia and performance under thermoneutral conditions. Food Funct 7, 872-880.

19. Gobatto CA, de Mello MA, Sibuya CY, et al. (2001) Maximal lactate steady state in rats submitted to swimming exercise. Comp Biochem Physiol A Mol Integr Physiol 130, 21-27.

20. Bough KJ, Valiyil R, Han FT, et al. (1999) Seizure resistance is dependent upon age and calorie restriction in rats fed a ketogenic diet. Epilepsy Res 35, 21-28.

21. Reeves PG, Nielsen FH \& Fahey GC Jr (1993) AIN-93 purified diets for laboratory rodents: final report of the American Institute of Nutrition ad hoc writing committee on the reformulation of the AIN-76A rodent diet. J Nutr 123, 1939-1951.

22. Contarteze RV, Manchado Fde B, Gobatto CA, et al. (2008) Stress biomarkers in rats submitted to swimming and treadmill running exercises. Comp Biochem Physiol A Mol Integr Physiol 151, 415-422.

23. Mesa J, Salcedo D, Calle Hde L, et al. (2006) Detection of ketonemia and its relationship with hyperglycemia in type 1 diabetic patients. Diabetes Res Clin Pract 72, 292-297.

24. Rogero MM, Tirapegui J, Pedrosa RG, et al. (2006) Effect of alanyl-glutamine supplementation on plasma and tissue glutamine concentrations in rats submitted to exhaustive exercise. Nutrition 22, 564-571.

25. Schlamowitz M (1951) On the nature of rabbit liver glycogen. II. Iodine absorption spectrum. J Bio Chem 190, 519-527.

26. Bowtell JL, Gelly K, Jackman ML, et al. (2000) Effect of different carbohydrate drinks on whole body carbohydrate storage after exhaustive exercise. J Appl Physiol (1985) 88, 1529-1536.

27. Cohen J (1992) A power primer. Psychol Bull 112, 155-159.

28. Czarnowski D, Langfort J, Pilis W, et al. (1995) Effect of a low-carbohydrate diet on plasma and sweat ammonia concentrations during prolonged nonexhausting exercise. Eur J Appl Physiol Occup Physiol 70, 70-74.
29. Wagenmakers AJ (1998) Muscle amino acid metabolism at rest and during exercise: role in human physiology and metabolism. Exerc Sport Sci Rev 26, 287-314.

30. Greenhaff PL, Leiper JB, Ball D, et al. (1991) The influence of dietary manipulation on plasma ammonia accumulation during incremental exercise in man. Eur J Appl Physiol Occup Physiol 63, 338-344.

31. Walser M (1975) Nutritional effects of nitrogen-free analogues of essential amino acids. Life Sci 17, 1011-1020.

32. Maddrey WC, Weber FL Jr, Coulter AW, et al. (1976) Effects of keto analogues of essential amino acids in portal-systemic encephalopathy. Gastroenterology 71, 190-195.

33. Snow RJ, Carey MF, Stathis CG, et al. (2000) Effect of carbohydrate ingestion on ammonia metabolism during exercise in humans. J Appl Physiol (1985) 88, 1576-1580.

34. Linnane DM, Bracken RM, Brooks S, et al. (2004) Effects of hyperthermia on the metabolic responses to repeated highintensity exercise. Eur J Appl Physiol 93, 159-166.

35. Li D, Wang X, Liu B, et al. (2014) Exercises in hot and humid environment caused liver injury in a rat model. PLOS ONE $\mathbf{9}$, e111741.

36. Halpin LE, Gunning WT \& Yamamoto BK (2013) Methamphetamine causes acute hyperthermia-dependent liver damage. Pharmacol Res Perspect 1, e00008.

37. Graham TE \& MacLean DA (1992) Ammonia and amino acid metabolism in human skeletal muscle during exercise. Can J Physiol Pharmacol 70, 132-141.

38. Harmer AR, McKenna MJ, Sutton JR, et al. (2000) Skeletal muscle metabolic and ionic adaptations during intense exercise following sprint training in humans. J Appl Physiol (1985) 89, 1793-1803.

39. Mohr M, Rasmussen P, Drust B, et al. (2006) Environmental heat stress, hyperammonemia and nucleotide metabolism during intermittent exercise. Eur J Appl Physiol 97, 89-95.

40. Febbraio MA (2001) Alterations in energy metabolism during exercise and heat stress. Sports Med 31, 47-59.

41. Morris JG, Nevill ME, Boobis LH, et al. (2005) Muscle metabolism, temperature, and function during prolonged, intermittent, high-intensity running in air temperatures of 33 degrees and 17 degrees C. Int J Sports Med 26, 805-814.

42. Savica V, Santoro D, Ciolino F, et al. (2005) Nutritional therapy in chronic kidney disease. Nutr Clin Care 8, 70-76.

43. Hellsten Y, Richter EA, Kiens B, et al. (1999) AMP deamination and purine exchange in human skeletal muscle during and after intense exercise. J Physiol 520, 909-920.

44. Parkin JM, Carey MF, Zhao S, et al. (1999) Effect of ambient temperature on human skeletal muscle metabolism during fatiguing submaximal exercise. J Appl Physiol (1985) 86, 902-908.

45. Banfi G, Colombini A, Lombardi G, et al. (2012) Metabolic markers in sports medicine. Adv Clin Chem 56, 1-54.

46. Baum N, Dichoso CC \& Carlton CE (1975) Blood urea nitrogen and serum creatinine. Physiology and interpretations. Urology $\mathbf{5}, 583-588$. 